\title{
Turbulence control by non-Hermitian potentials
}

\author{
Salim B. Ivars ${ }^{1}$, Muriel Botey ${ }^{1}$, Ramon Herrero ${ }^{1}$, Kestutis Staliunas ${ }^{1,2}$ \\ 1. Universitat Politècnica de Catalunya (UPC), 08222, Terrassa, Barcelona, Catalonia, Spain \\ 2. Institució Catalana de Recerca i Estudis Avançats (ICREA), 08010, Barcelona, Catalonia, Spain
}

We propose a method to control turbulence by the introduction a non-Hermitian spatiotemporal modulation, based on the energy redirection from unstable to stable modes by the unidirectional coupling induced by these potentials and so, influence and slow down the excitation cascade of turbulence. The phenomenon of the turbulence is, and has been for a long time an interesting and challenging topic for scientist. Plenty of turbulent systems follow a quite general scenario, the excitation in a continuous system imposed on a large spatial scale, decreases in scale due to nonlinear wave-mixing processes until finally entering into a dissipative range, on a small spatial scale. Turbulence bridges these two spatial scales and results in an energy cascade through the wavenumbers. Turbulence appears in a wide range of disciplines, from fluids to business and optics. For this last case, turbulence represents one of the main inconvenience for laser source. These sources loss stability when power is increased through a instability of spatial character, the Modulation Instability (MI). Some techniques have been proposed to reduce and suppress the MI [1]. Aside, the introduction of non-Hermitian potentials has shown an asymmetric or even unidirectional coupling between modes allowing different spatial effects [2-3].

In this work we numerically analyze the turbulence spectrum which can be modeled in the universal Complex Ginzburg Landau Equation (CGLE) with a non-Hermitian spatiotemporal modulation in the form:

$$
V(x, t)=\cos (q x)\left[m 1 \cos (\Omega t)+i m 2 \cos \left(\Omega t+\phi_{t}\right)\right] .
$$

The spatial sinusoidal modulation introduced by the potential breaks the spatial symmetry while the complex temporal modulation with a relative phase shift between real and imaginary parts induce a directional coupling between spectral modes. Such potential may induce different couplings in the turbulent spectrum of the unmodulated CGLE, following the linear dispersion $\omega !=k_{x}$ (as represented by the arrows 1,2,3, and 4 in Fig. 1(a) for the $1 \mathrm{D}$ case)). Time averaged 2D turbulent spectrum is depicted in Fig. 1(b). Depending on the sign of the temporal frequency modulation $\Omega$ ! some of those couplings are enhanced (either those labelled as 1 and 2 for $\Omega<$ 0 , or 3 and 4 for $\Omega>0$ ). The first ones favor couplings towards lower order modes and, as a consequence, the energy cascade accumulates the spectrum intensity near $k_{x}=0$, thus reducing the turbulence as shown in Fig. 1(c). Alternately, in the second case, energy spreads to high $k_{x}$ values, increasing turbulence.

The phase shifts of the temporal potential can either increase or reduce turbulence. We note that we have observed a $\pi / 2$ shift from the conventional PT-symmetric theory that was developed for only spatial nonHermitian potentials, which can be analytically understood.
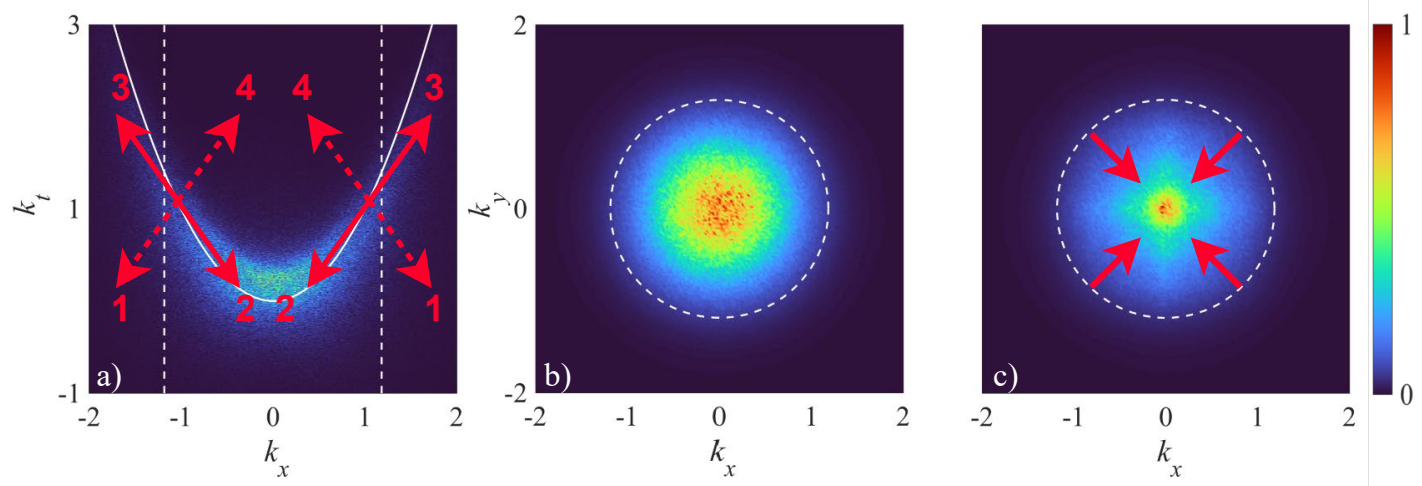

Fig. 1 a) 1D turbulent normalised spectrum amplitude. The white parabola corresponds to the linear dispersion $\omega !=k x 2$. The arrows indicate all possible mode couplings. b) and c) show the normalised time averaged 2D turbulent spectrum for the unmodulated and modulated CGLE. 
We prove the idea for one- and two-dimensional systems with several spatial modulation arrangements such as a linear, square grid, hexagonal and radial configurations. Indeed, we show that periodic potentials in space with non-Hermitian modulation in time may provide a new turbulence reduction method.

\section{References}

[1] Kumar, S., Herrero, R., Botey, M., \& Staliunas, K. (2015). "Taming of modulation instability by spatio-temporal modulation of the potential". Sci rep, 5(1), 1-7.

[2] Bender, C. M., \& Boettcher, S. (1998). "Real spectra in non-Hermitian Hamiltonians having P T symmetry”. Phys Rev Lett, 80 (24), 5243.

[3] Ahmed, W. W., Herrero, R., Botey, M., \& Staliunas, K. (2017). "Self-collimation in P T-symmetric crystals". Phys Rev A, 95(5), 053830. 